\title{
Glass patterns in the superposition of random line gratings
}

\author{
Isaac Amidror \\ Laboratoire de Systèmes Périphériques (LSP), Ecole Polytechnique Fédérale de Lausanne \\ (EPFL), 1015 Lausanne, Switzerland
}

Received 20 June 2002, in final form 7 January 2003

Published 28 March 2003

Online at stacks.iop.org/JOptA/5/205

\begin{abstract}
Moiré effects ('Glass patterns') that occur in the superposition of correlated random layers have long been studied. However, only little is known of Glass patterns which occur in the 1D random case: namely, when the superposed layers consist of random line gratings, straight or curved. In this paper we study the properties and the behaviour of such Glass patterns, and we compare them with those of the analogous moiré effects between periodic line gratings. We provide for each case a detailed mathematical analysis of the fringe shapes and locations, along with illustrative figures which clearly compare the behaviour of the corresponding random and periodic (or repetitive) superpositions.

Publisher's note. We believe the moiré images in this article will print faithfully from the online pdf file but some spurious fringing will probably occur on-screen, depending on the screen resolution. To minimize that effect, we recommend viewing on-screen at $200 \%$ or higher magnification in Acrobat or Acrobat Reader.
\end{abstract}

Keywords: Glass patterns, stochastic moiré, periodic moiré, line gratings, superposition

\section{Introduction}

When two identical random dot screens, or any two identical 2D random structures, are superposed on each other with a small angle or scaling difference, a typical moiré effect having the shape of a top-viewed funnel appears in the superposition (see figure 1(b)). This moiré effect is known in the literature as a Glass pattern, named after Leon Glass who described it in the late 1960s [1, 2], Unlike a moiré effect between periodic layers (figure 1(d)), which is periodic and extends throughout the entire superposition [3], a Glass pattern is concentrated around a certain point in the superposition, and it gradually fades away and disappears as we move further away from this point.

A similar phenomenon also occurs in the 1D case: namely, where the original layers consist of line gratings. However, although Glass patterns which occur in the 1D case differ from their $2 \mathrm{D}$ counterparts in several respects, their properties have not previously been elucidated.

In this paper we investigate Glass patterns in the superposition of random line gratings, either straight or curved.
We compare them with their 2D counterparts, and explain the significant differences between them. We also compare their behaviour with that of the moiré patterns which are obtained in superpositions of periodic or repetitive line gratings, and we explain the similarities as well as the differences between them. We provide the mathematical derivation of their curve shapes and locations, along with illustrative figures that clearly compare their behaviour with that of their corresponding periodic counterparts.

In section 2 we explain the basic phenomena which are related to the superposition of aperiodic layers. In section 3 we discuss the Glass patterns which are generated in the superposition of straight line gratings, and we explain how such cases can be analysed mathematically. Then, in section 4 we extend our discussion to Glass patterns in the superposition of curved line gratings. Finally, in section 5 we present the main conclusions.

Remark. The PostScript files that generate the line gratings used in the figures of this paper are available on the internet ${ }^{1}$.

\footnotetext{
1 The PostScript files have been added at the end of the Moiré Demonstration Kit located at the Internet address http://lspwww.epfl.ch/books/moire/kit.html.
} 


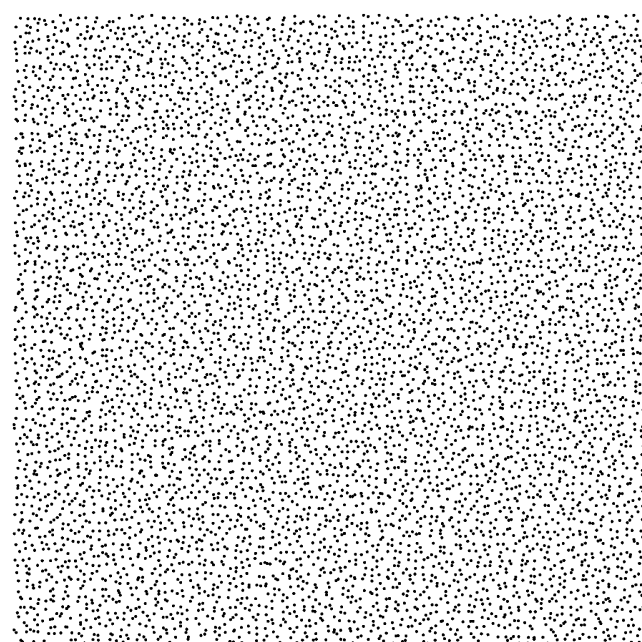

(a)

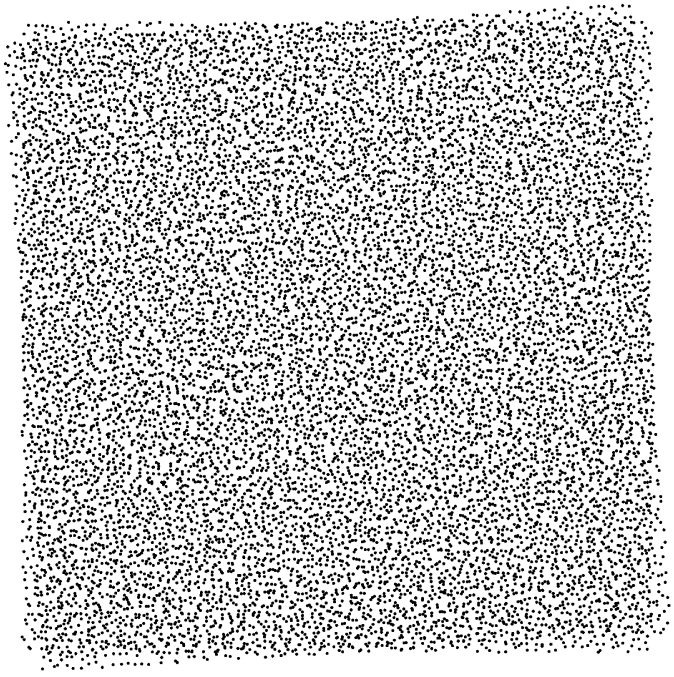

(c)

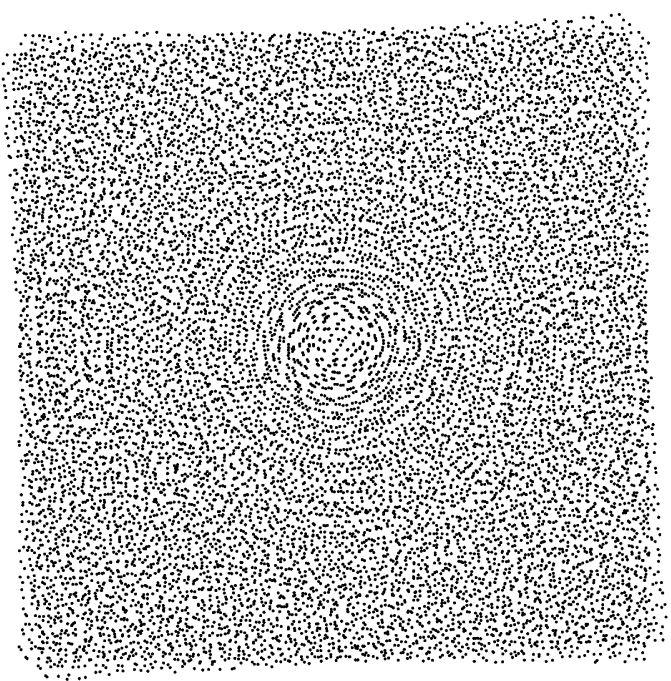

(b)

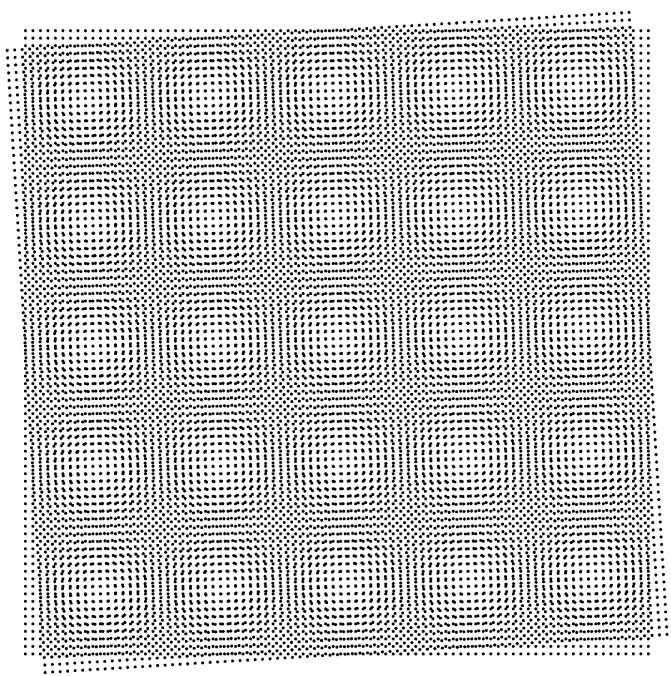

(d)

Figure 1. (a) An aperiodic dot screen. (b) The superposition of two identical copies of the aperiodic dot screen (a) with a small angle difference gives a moiré effect in the form of a Glass pattern around the centre of rotation. (c) When one of the aperiodic layers is placed face down on top of the other layer, the Glass pattern completely disappears. (d) When the superposed layers are periodic, a Glass pattern is still generated around the centre of rotation, but due to the periodicity of the layers, this pattern is periodically repeated throughout the superposition, thus generating a periodic moiré pattern.

They can be downloaded and printed on transparencies using any standard PostScript printer. Superposing these transparencies manually with varying orientations, shifts, etc can give a vivid demonstration of the Glass (or moiré) patterns and their dynamic behaviour in the superposition, beyond the few static figures that illustrate this paper.

\section{Glass patterns in the superposition of $2 \mathrm{D}$ aperiodic layers}

While the superposition of two similar periodic layers generates moiré effects that are themselves periodic, the superposition of two similar 2D aperiodic layers (such as random dot screens, etc) generates an aperiodic moiré effect known as a Glass pattern (see figure 1(b)). This moiré pattern is concentrated around a certain point in the superposition, and in contrast to periodic moirés, it gradually disappears as we move further away from this point. Depending on whether it was obtained by rotation of one of the superposed layers, by a scaling transformation, or by a combination of both, it gives rise to an intriguing ordering of the microstructure elements in the superposition in 'trajectories' having a circular, radial or spiral shape (figures 1(b), 2(a), 2(b)) [2]. Other layer transformations may give rise to Glass patterns having elliptic, hyperbolic or other geometrically shaped trajectories [2]. However, when we place one of the superposed aperiodic layers face down on top of the other layer (this is easy to do when experimenting with transparencies; see figure 1(c)), the Glass pattern disappears as if by magic.

As already explained by Glass, this phenomenon occurs thanks to the local correlation between the structures of the two superposed layers. When two identical layers having the same arbitrary structure are slightly rotated on top of each other (figure 1(b)), a visible Glass pattern is generated around the centre of rotation, indicating the high correlation between the two layers in this area: within the centre of the Glass pattern the 


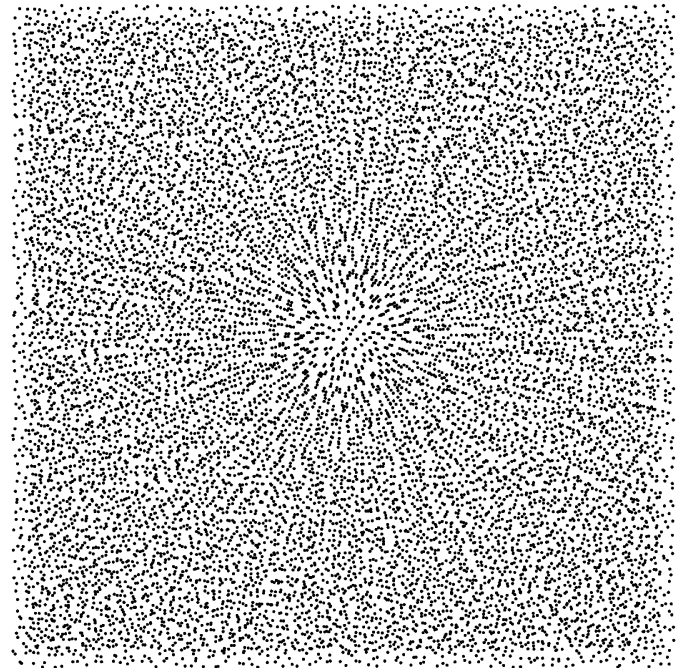

(a)

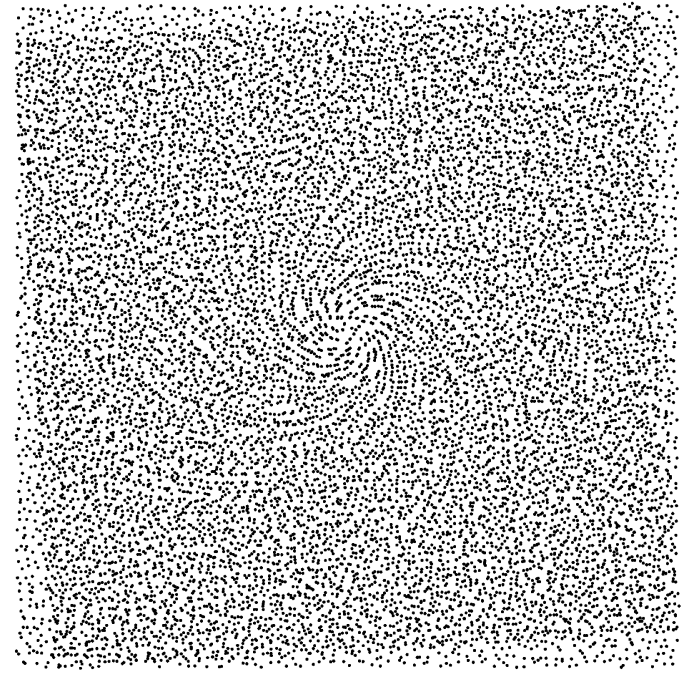

(b)

Figure 2. (a) As figure 1(b), but with a small scaling difference (rather than an angle difference) between the two layers; note that in this case the microstructure consists of radial trajectories rather than concentric circular trajectories. (b) As in (a), but with both a small angle and a small scaling difference between the two identical layers; note that in this case the microstructure consists of spiral trajectories.

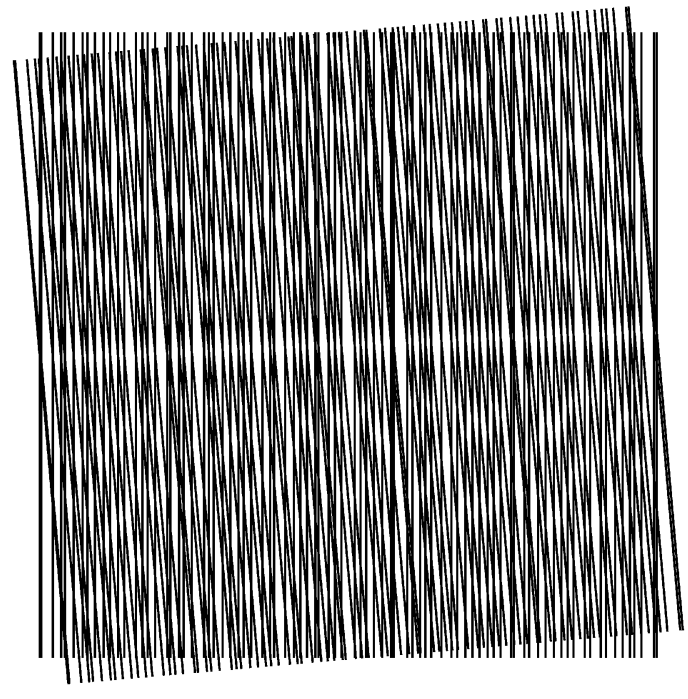

(a)

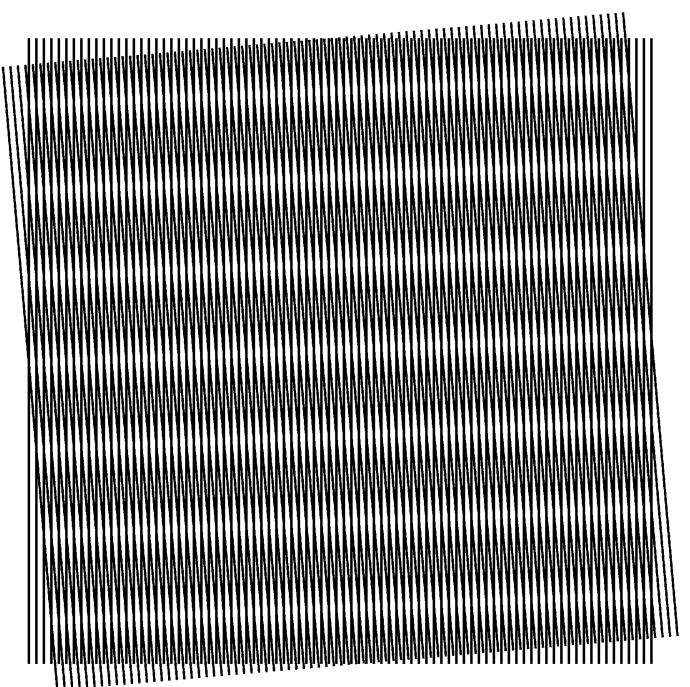

(b)

Figure 3. Glass patterns in the superposition of straight line gratings. (a) The superposition of two identical aperiodic line gratings with a small angle difference of $\alpha=5^{\circ}$. (b) The superposition of two identical periodic line gratings with the same angle difference. In both (a) and (b) a linear Glass pattern is generated precisely at the same location.

corresponding elements from both layers fall almost exactly on top of each other, but slightly away from the centre they fall just next to each other, generating circular trajectories of point pairs. Further away from the centre the correlation between the two layers becomes smaller and smaller, and the elements from both layers fall in an arbitrary, non-correlated manner; in this area the Glass pattern is no longer visible. This explains why the Glass pattern gradually decays and disappears as we move away from its centre. Note, however, that when the two superposed layers are not at all correlated, no Glass pattern appears in the superposition (this is, indeed, what happens when we place one of the aperiodic transparencies face down on top of its identical copy, as shown in figure 1(c)). In intermediate cases, where the two superposed layers are only partially correlated (for example, when one layer is a copy of the other with some per cent of random noise being added), the
Glass pattern becomes weaker and less perceptible, depending on the degree of the correlation which still remains between the superposed layers.

The explanation above is based on an observation of the individual elements of the original layers and their behaviour in the superposition. We say, therefore, that this explanation is based on the microstructure. To obtain the point of view of the macrostructure, we have to look at the layers and their superposition from a greater distance, where the individual elements of the layers are no longer discerned by the eye and what we see is only a grey level average of the microstructure in each area of the superposition. From the point of view of the macrostructure, the centre of the Glass pattern consists of a lighter grey level than areas further away, due to the partial overlapping of the microstructure elements of both layers in this area. Slightly away from the centre elements 
from the two layers are more likely to fall side by side, thus increasing the covering rate and the macroscopic grey level. But as we move further from the centre the correlation between the two layers becomes smaller, and the elements from both layers start falling in an arbitrary, non-correlated manner; in this area the Glass pattern fades away, and we obtain a mean grey level which remains constant throughout. This means that the Glass pattern is not just an optical illusion, but corresponds to the physical reality. In fact, just as in the periodic case (see proposition 8.1 in [3]), moiré patterns are simply the macroscopic interpretation of the variations in the microstructures throughout the superposition.

As we have seen, the superposition of layers, periodic or not, gives rise to a moiré pattern iff there exists some degree of correlation between the superposed structures. Therefore, in order to study the behaviour of a Glass (or moiré) pattern, we have to make sure that the layers we superpose are sufficiently correlated. Clearly, the easiest way of doing so is to assume that the superposed layers, periodic or not, were identical before the application of the layer mappings (rotation, scaling, etc). This also implies, in the case of random layers, the use of the same seed for the random number generator in both layers. In the following we make this assumption. This does not cause a loss of generality, since in cases where the original layers are only partially correlated (for example: due to the presence of some random noise), the Glass patterns are simply less visible, but their behaviour under layer transformations remains the same.

\section{Glass patterns in the superposition of straight line gratings}

Having understood the behaviour of Glass patterns in the 2D case (superpositions of dot screens, etc), we are ready now to proceed to the phenomena which occur in the 1D case, i.e. where the original layers (periodic or not) consist of line gratings $^{2}$. The $1 \mathrm{D}$ case is conceptually simpler than the $2 \mathrm{D}$ case that we have discussed so far, due to its inherently limited structural complexity. As we will see in the examples below, even the dot trajectories, which are among the most striking features of Glass patterns in 2D cases, are practically absent in $1 \mathrm{D}$ cases. Yet the investigation of the 1D case is no less interesting than that of the 2D case, and in fact yields some surprising results.

\subsection{Observations}

We start with the superposition of two identical straight random gratings with a small angle difference $\alpha$. Unlike in the superposition of two identical random dot screens with a small angle difference, where a circular Glass pattern is generated around the origin (figure 1(b)), in the present case a linear Glass pattern is generated in the layer superposition along a given straight line passing through the origin (figure 3(a)). This line is, in fact, the locus of the points of coincidence between the two superposed layers. Along this line the correlation between

2 Use of the term '1D grating' is, strictly, an abuse of language, since in fact this is still a 2D layer. The more appropriate term that should be understood here is 'a 1D structure which has been constantly extended perpendicularly to its main direction within the $2 \mathrm{D}$ plane'. the superposed layers is maximal, since the black and white microstructure elements of both layers fall exactly on top of one another. But as we move further from this line the correlation between the layers gradually decreases: the elements of both layers gradually start falling arbitrarily between each other, and the Glass pattern fades away. Note that just as in the circular Glass pattern between two dot screens, the centre of the linear Glass pattern is brighter than its surrounding area. This is, indeed, the macroscopic consequence of the fact that in the centre of the linear Glass pattern microstructure elements fall exactly on top of each other, while farther away they start falling between each other, so that less white area is left and the superposition looks darker. Far away from the linear Glass pattern there is no longer any correlation between the two layers, and we obtain a mean grey level which remains constant throughout. This macroscopic view is best appreciated by observing the layer superpositions from a sufficient distance (say, 3-4 m), where the individual elements of the layers are no longer discerned by the eye and what we see is only a grey level average of the microstructure in each area of the superposition.

Let us see now what happens in the superposition when we slightly modify the angle difference $\alpha$ between the two identical line gratings. As shown in figure 4(a), when $\alpha$ increases, the linear Glass pattern becomes thinner, and its angle is slightly increased (in fact, as we will see in the mathematical derivation below, its orientation is perpendicular to the bisector between the main directions of the two superposed line gratings). When $\alpha$ tends to zero, the linear Glass pattern becomes wider and wider, and approaches the angle of zero. And when $\alpha=0$, i.e. when the two superposed gratings precisely coincide, the width of the linear Glass pattern becomes infinite, and it completely disappears.

Next, let us see what happens in the superposition when we also scale one of the superposed line gratings. As we can see by comparing figures 3(a) and 5(a), when the scaling ratio $s$ departs from 1 the linear Glass pattern becomes thinner and its orientation is modified.

Finally, let us see what happens when we slightly shift one of the superposed line gratings on top of the other along its main direction. As we can see by comparing figures 3(a) and 6(a), the linear Glass pattern in the superposition will undergo a much larger shift, while its angle remains unchanged. A layer shift along the secondary direction, namely, along the lines of the grating, will obviously have no effect on the superposition.

\subsection{Superposition of $1 D$ versus $2 D$ random layers}

As we can see, there exists a significant difference between the Glass patterns which are generated between random line gratings and the Glass patterns between random dot screens: while the classical Glass patterns between random dot screens are generated around a point of coincidence between the superposed layers, the linear Glass patterns between random line gratings are generated along a full 1D line of coincidence between the superposed layers (compare, for example, figure 1(b) with 3(a) or 2(b) with 5(a)). The reason for this difference is explained as follows.

In a superposition of two identical dot screens one of which has been slightly rotated or scaled, the Glass pattern is generated around the fixed point of the transformation 


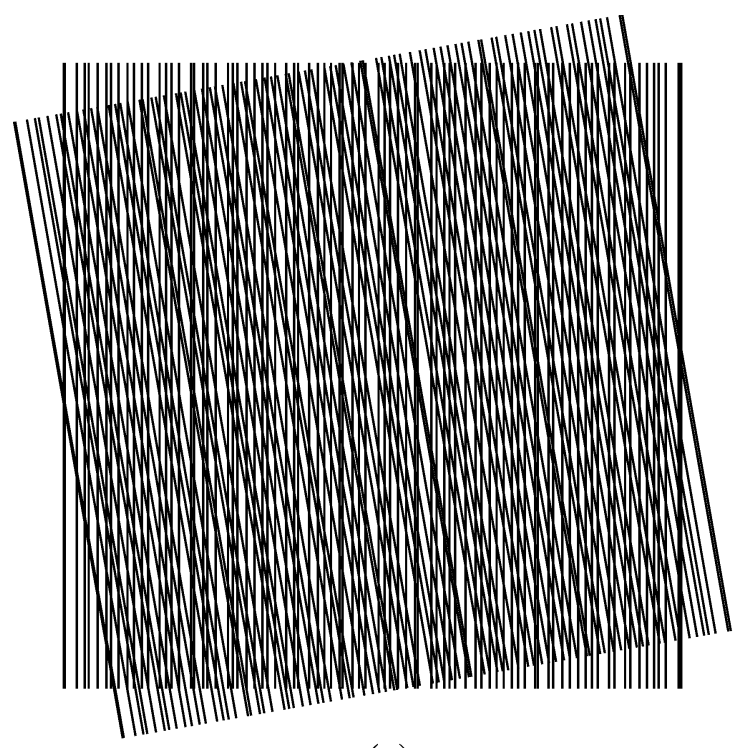

(a)

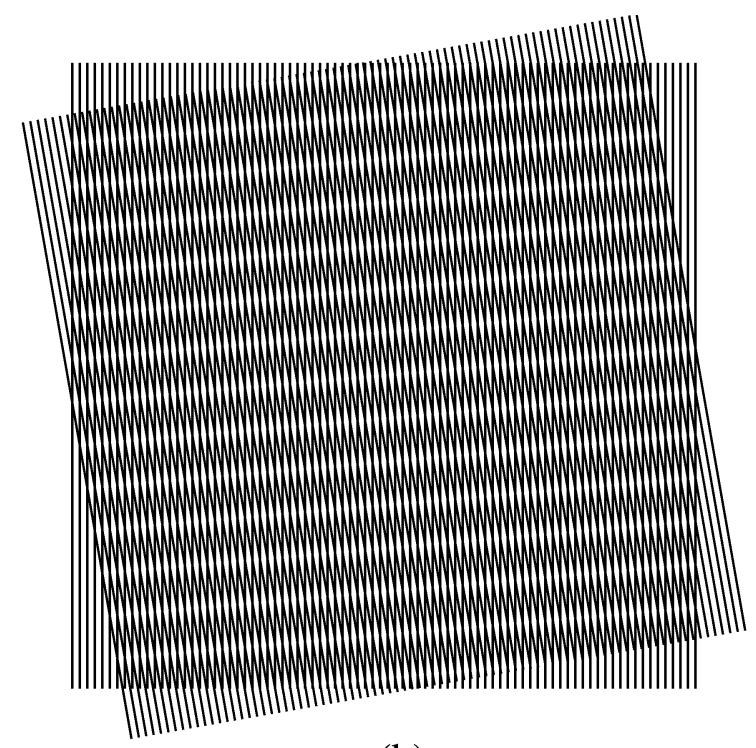

(b)

Figure 4. As figure 3, but with $\alpha=10^{\circ}$.

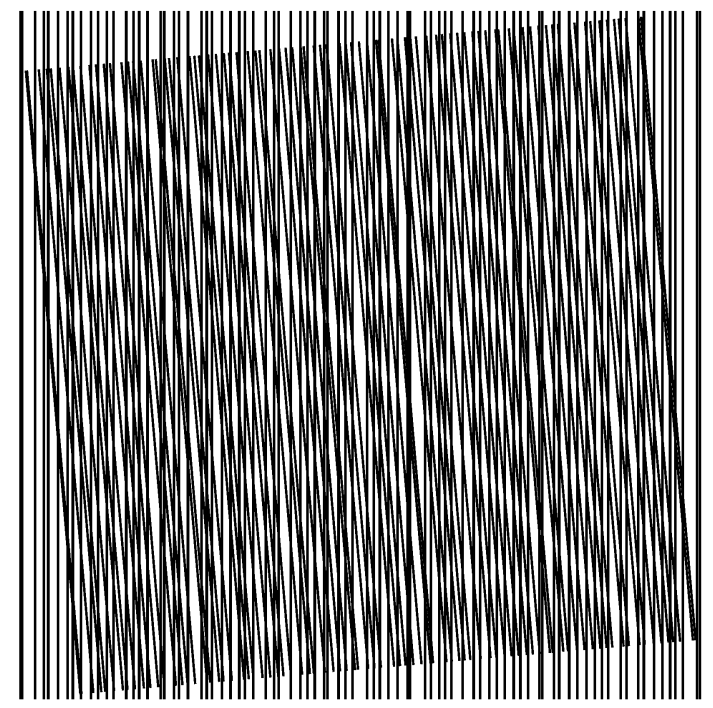

(a)

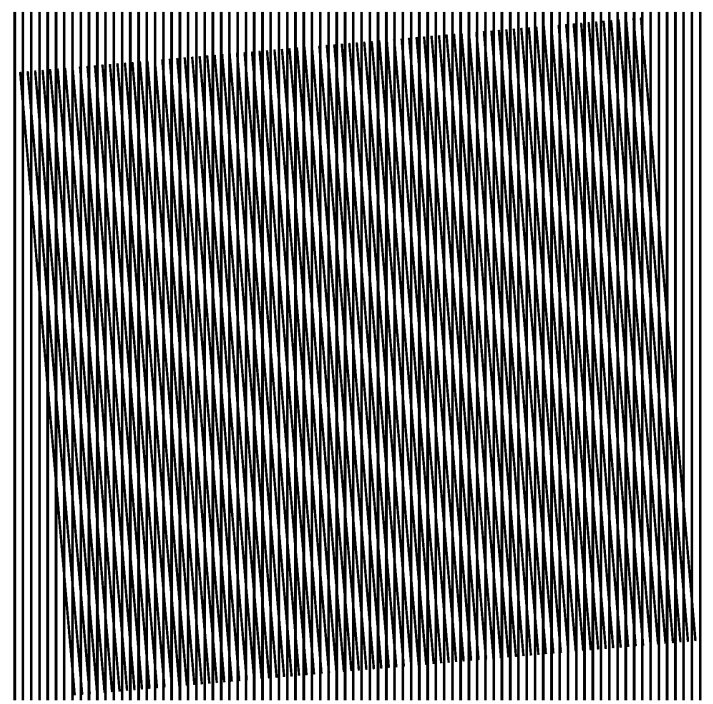

(b)

Figure 5. As figure 3, but here the unrotated layer has been slightly scaled by $s=1.1$.

$\boldsymbol{g}(x, y)$ in question, namely: the point $\left(x_{F}, y_{F}\right)$ for which $\boldsymbol{g}\left(x_{F}, y_{F}\right)=\left(x_{F}, y_{F}\right)$. Any rotation or scaling transformation $\boldsymbol{g}(x, y)$ has exactly one such fixed point.

Suppose now that we superpose, instead of random dot screens, two identical random line gratings, and that we apply on one of them exactly the same transformation $\boldsymbol{g}(x, y)$ as in the case of the dot screens above: namely, a slight rotation or scaling. Obviously, in both cases the underlying transformation $\boldsymbol{g}(x, y)$ has exactly one and the same fixed point. But when the two superposed layers are line gratings, there also exist between the two superposed layers infinitely many points of coincidence, which together form a full straight line passing through the real fixed point. The two superposed gratings coincide along this line because of the 1D nature of their internal structure. Indeed, a random line grating is more constrained (i.e. it has fewer degrees of freedom) than a random dot screen, since it only has full freedom in one dimension, while its other dimension is fully determined (constant). Therefore, due to the additional restriction on the structure of the layers (namely, the fact that their second dimension is fully determined by the first one), the layer superposition has a full line of points of coincidence (which obviously includes the fixed point of the underlying mapping).

We can therefore formulate our result as follows: Glass patterns in the superposition of random gratings are not only generated around the fixed points of the underlying layer transformations, as in the 2D case, but they extend into full lines. In fact, as we will see in section 4, these lines may even be curved, when the superposed layers consist of curved line gratings.

\subsection{Superposition of random versus periodic line gratings}

Figures 3-6 show side by side the behaviour of the linear Glass pattern in the superposition of two random line gratings (panel 


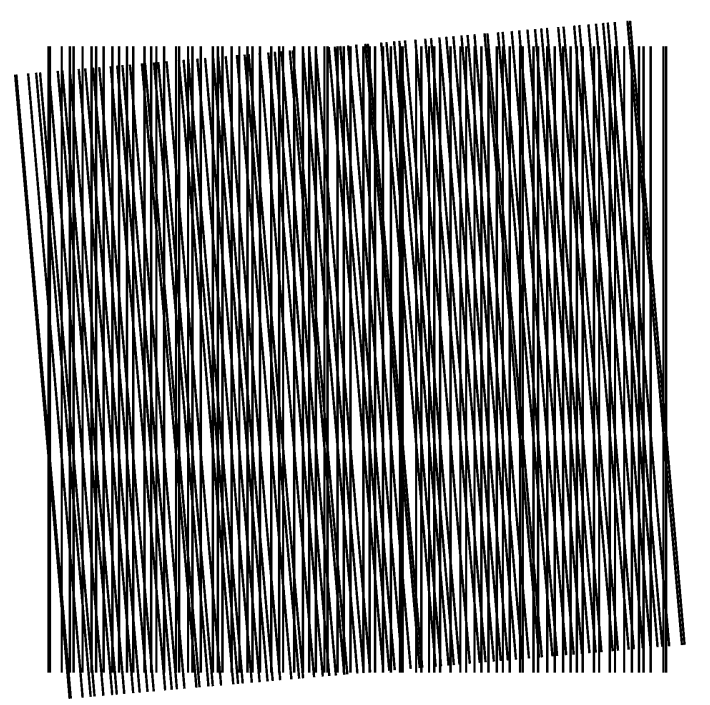

(a)

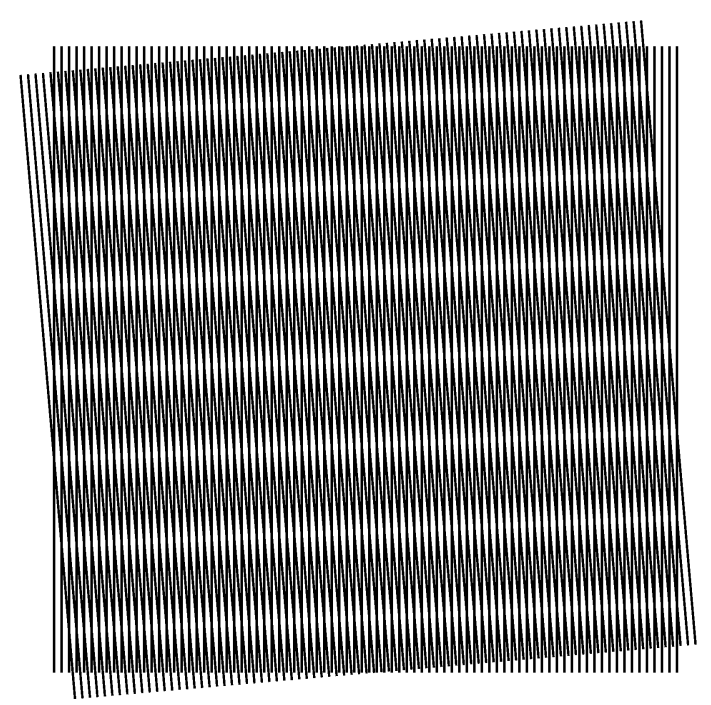

(b)

Figure 6. As figure 3, but here the unrotated grating has been slightly shifted horizontally by $x_{0}=T$, where $T$ equals one period of the periodic grating of $(b)$.

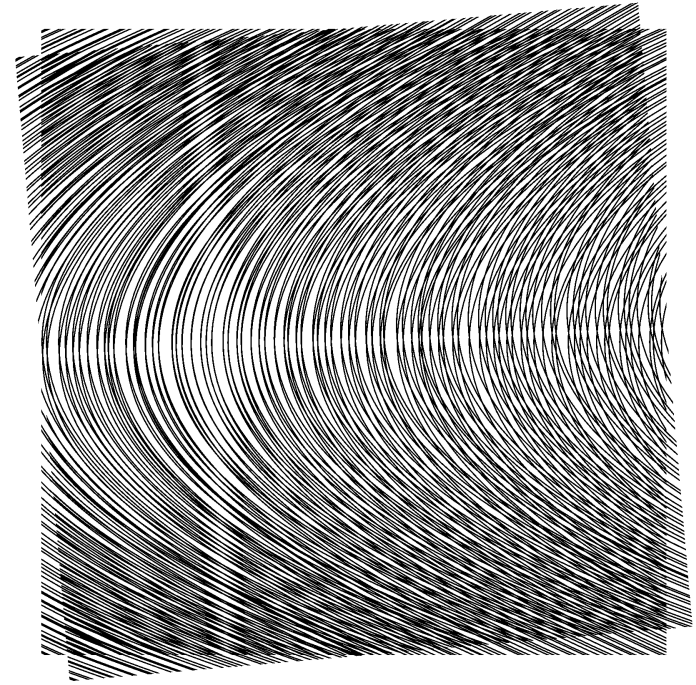

(a)

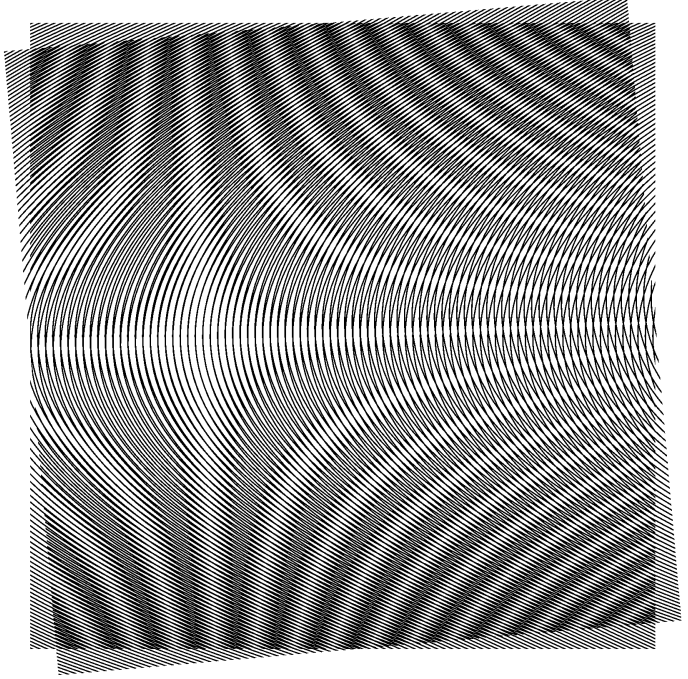

(b)

Figure 7. Glass patterns in the superposition of parabolic line gratings. (a) The superposition of two identical aperiodic parabolic line gratings with a small angle difference of $5^{\circ}$. (b) The superposition of two identical periodic parabolic line gratings with the same angle difference. In both (a) and (b) two perpendicular linear Glass patterns are generated precisely at the same location.

(a) of each figure), and the behaviour of the moiré patterns in the superposition of a corresponding pair of periodic line gratings having undergone exactly the same rotations, scalings and layer shifts (panel (b) of each figure). As we can clearly see from these figures, in both aperiodic and periodic cases a linear Glass pattern is generated exactly in the same location, along the line of coincidence between the superposed gratings. Note that this line passes through the fixed point of the underlying layer transformation $\boldsymbol{g}(x, y)$. However, while the aperiodic moiré consists of only one linear Glass pattern, the moiré effect in the corresponding superposition of periodic gratings consists of infinitely many repetitions of this Glass pattern. In other words, the Glass pattern which is generated along the line of coincidence is periodically repeated throughout the superposition, forming the bright bands of the periodic moiré pattern. From this point of view, the periods of the periodic moiré pattern are simply duplicates of the main linear Glass pattern which is generated through the fixed point, and the period length of the moire corresponds to the distance between these duplicates ${ }^{3}$. This does not mean, of course, that our rotation or scaling transformation $g(x, y)$ has more fixed points when the two superposed layers are periodic than when the layers are aperiodic: obviously, in both cases $\boldsymbol{g}(x, y)$ has exactly one fixed point. But unlike in the case of 2D aperiodic layers, such as random screens, in the superposition

3 It is important to note, however, that these duplicates are not necessarily identical in their microstructure, and the periodicity of the moiré concerns only its macrostructure: namely, the moiré intensity profile (the variation in the mean grey level that is observed from such a distance that the microstructure detail of the original layers is no longer discerned by the eye). In other words, although the microstructure in the superposition of two periodic layers is not always periodic, the intensity profile of the isolated moire is, indeed, periodic (see section 6.3 in [3]). 
of line gratings this fixed point is no longer the only point of coincidence between the superposed layers. As we saw in section 3.2, the superposition of identical gratings with a small angle difference already gives a full line of coincidence between the two superposed layers, which includes the fixed point of $\boldsymbol{g}(x, y)$. But when the two superposed layers are also periodic, we have, in addition, infinitely many new lines of coincidence between the two superposed layers, where the two layers happen to coincide because of the periodicity in their internal structure. These new lines of coincidence are not fixed points of the underlying mapping $\boldsymbol{g}(x, y)$, nor coincidence lines in the case of random gratings. We can say, therefore, that the fixed point of $\boldsymbol{g}(x, y)$ determines the main periodic band (or Glass pattern) of the moiré, while all the other moiré bands that are generated in the periodic case are only duplicates which exist due to the periodicity of the superposed layers.

Hence, as we have already seen for $1 \mathrm{D}$ versus $2 \mathrm{D}$ cases in section 3.2, the fact of having additional constraints on the internal structure of the superposed layers (here: due to their periodicity) causes the addition of new loci of coincidence in the superposition, which are not loci of coincidence in the corresponding superposition of unconstrained layers. Having constrained (fixed) the second dimension of the superposed layers results in the extension of their locus of coincidence from the fixed point of the underlying transformation $g(x, y)$ into a full line; and adding the constraint of periodicity on the superposed layers causes the extension of this line into a family of infinitely many periodic lines of coincidence.

Looking at this phenomenon the other way around, we can say that by applying the same layer transformation $g(x, y)$ on aperiodic gratings instead of on periodic gratings, we can select one of the infinitely many repetitions of the Glass patterns which from together the repetitive macroscopic moiré effect. Which one exactly is selected depends on the precise shifts of the original layers, i.e. on their relative positioning. Note, however, that this property concerns only the macrostructures, since the microstructure within these elements may vary depending on the case. This interesting result is clearly illustrated by figures 3-6.

Let us now proceed to the mathematical derivation of the points of coincidence in the superposition of two straight line gratings. We will illustrate this derivation for the case with a small angle difference $\alpha$. Our first line grating is a family of vertical lines that is defined by

$$
x=c_{i} \quad i \in \mathbb{Z}, c_{i} \in \mathbb{R} .
$$

Our second line grating is a copy of the first line family which has been rotated by angle $\alpha$ :

$$
x \cos \alpha+y \sin \alpha=c_{i} \quad i \in \mathbb{Z}, c_{i} \in \mathbb{R} .
$$

This gives us a system of two equations in $x$ and $y$, whose solutions $(x, y)$ define the locus of the points of coincidence between the two layers. Note that $c_{i}$ are arbitrary numbers which are identical in both line families; this is, indeed, the key point which guarantees the correlation between the two layers. Since $c_{i}$ are identical in both line families, we can eliminate them from our two equations. We obtain, therefore,

$$
x \cos \alpha+y \sin \alpha=x
$$

which gives

$$
y=x \frac{1-\cos \alpha}{\sin \alpha}
$$

or, using the identity $\frac{1-\cos \alpha}{\sin \alpha}=\tan \frac{\alpha}{2}$,

$$
y=x \tan \frac{\alpha}{2} .
$$

The solution of our system of equations is, therefore, a straight line through the origin having the angle of $\frac{\alpha}{2}$. This is, indeed, the locus of the points of coincidence between the two layers, i.e. the centre of the linear Glass pattern (see figure 3(a)). Note that in the periodic case (figure 3(b)) the arbitrary numbers $c_{i}$ are simply replaced in equations (1) and (2) by integer multiples of the grating periods, $m T_{1}$ and $n T_{2}$, respectively. As explained in [3] (pp 354-355), this gives us the family of moiré bands which are generated in the superposition of the two periodic gratings. Note that the Glass pattern we have derived above is simply the 0th moiré band of this family, which is obtained when $m=n$ (namely, the moiré band whose index is $p=m-n=0$ ).

A similar derivation can be also done for cases which include layer shifts and layer scalings.

\section{Glass patterns in the superposition of curved line gratings}

When we apply a non-linear geometric transformation on an initially periodic straight line grating we obtain a curved repetitive grating. Moiré effects that occur between curved repetitive gratings have been intensively investigated in the past, and their mathematical theory is today fully understood (see, for example, chapters 10 and 11 in [3]).

Our aim in this section is to study the behaviour of Glass patterns between curved random line gratings: namely, straight random line gratings which have undergone a nonlinear mapping $\boldsymbol{g}(x, y)$. As an illustration to such curved line gratings we use here parabolic gratings. These curved gratings offer us three main advantages: they clearly illustrate the main configurations which occur in the case of curved gratings; their mathematical handling is still tractable; and finally, the results for the repetitive case (i.e. where the original uncurved gratings were periodic) are already known (see [3], sections 10.7.310.7.4), which enables us to compare the results obtained for periodic and aperiodic layers 4 .

We start by superposing two identical copies of our parabolic grating, origin on origin, and we apply to one of them a slight rotation of angle $\alpha$. As we can see in figure 7(a), the locus of the points of coincidence in this case consists of two perpendicular straight lines and, indeed, a Glass pattern is generated in the superposition along these lines.

But as we might expect, based on our experience with straight gratings, the superposition in the repetitive case contains, in addition to this Glass pattern, infinitely many other hyperbolic lines of coincidence between the two layers, which are not lines of coincidence in the aperiodic case (compare figures 7(a) and (b)). This happens due to the additional

4 Note that we have chosen horizontally oriented parabolas in order to remain compatible with the figures and the examples given in sections 10.7.3-10.7.4 of [3], some of which are used here, too. 


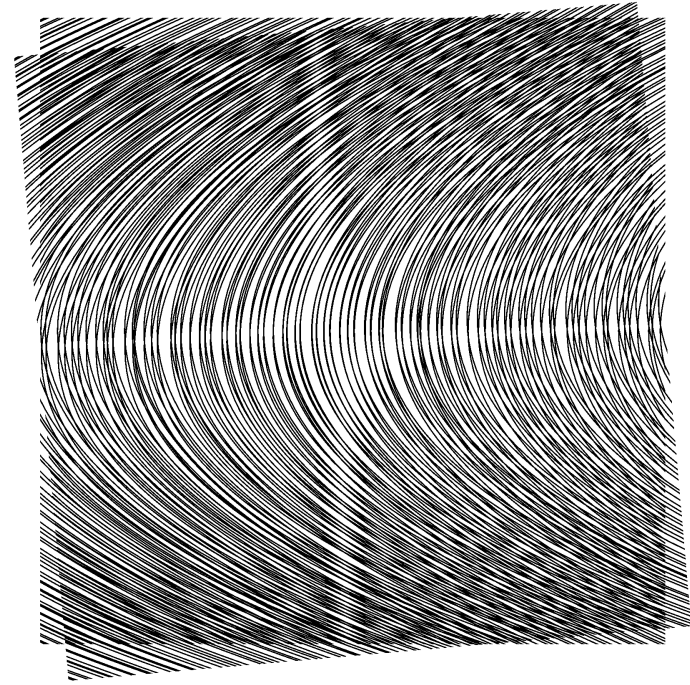

(a)

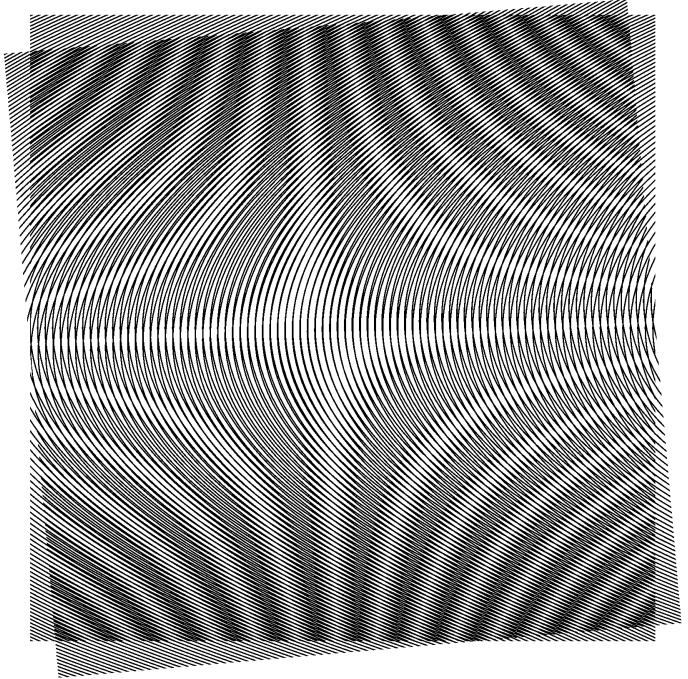

(b)

Figure 8. As figure 7, but the unrotated grating has been slightly shifted vertically by $y_{0}=1.5 T$, where $T$ equals one period of the original (untransformed) line gratings of (b). In both (a) and (b) two perpendicular linear Glass patterns are generated precisely at the same location.

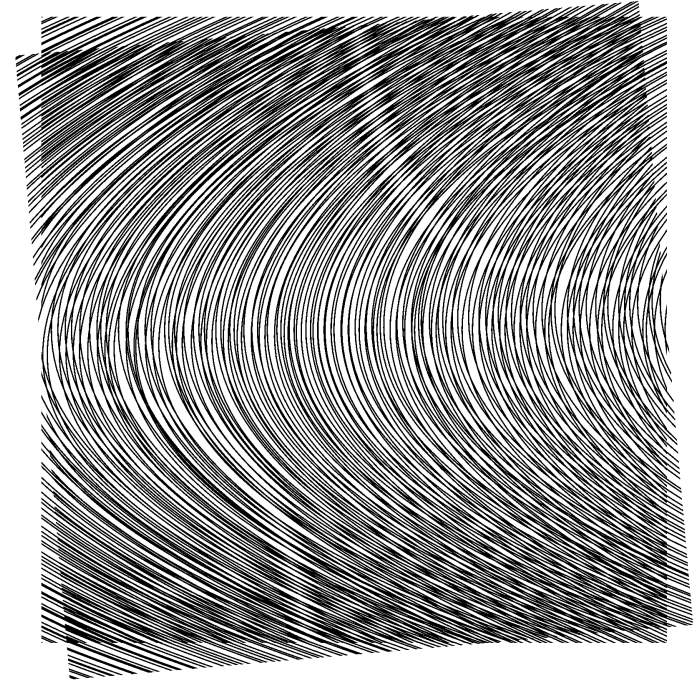

(a)

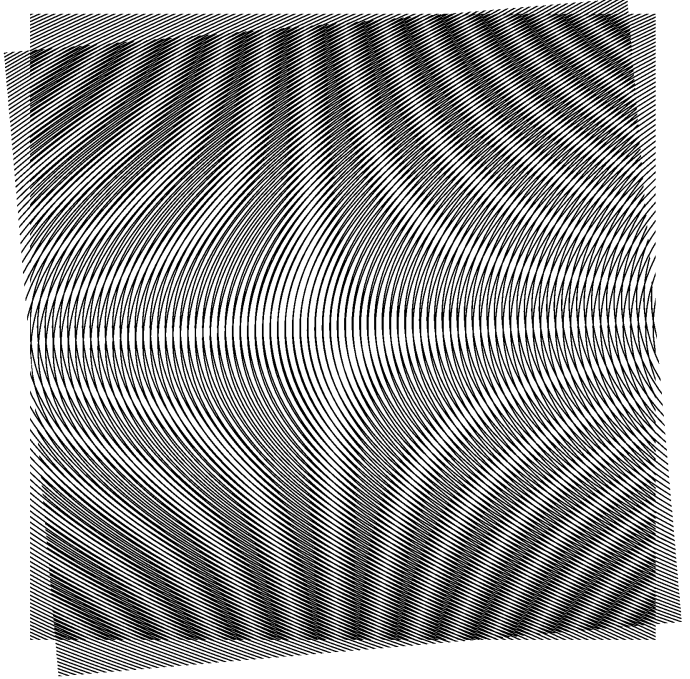

(b)

Figure 9. As figure 7, but with a slight layer shift of $\left(x_{0}, y_{0}\right)=(T, 1.5 T)$, where $T$ equals one period of the original (untransformed) line gratings of (b). In both (a) and (b) a hyperbolic Glass pattern is generated precisely at the same location.

ordering which exists in the internal structure of repetitive layers: thanks to the new constraints imposed by their repetitivity, the superposed layers also have infinitely many new lines of coincidence which are not lines of coincidence in the corresponding aperiodic case.

Let us now derive mathematically the points of coincidence in this superposition of two parabolic gratings. Our first grating is a family of parabolic lines that is defined by

$$
x-a y^{2}=c_{i} \quad i \in \mathbb{Z}, c_{i} \in \mathbb{R} .
$$

Our second grating is a copy of the first line family which has been rotated by angle $\alpha$ :

$x \cos \alpha+y \sin \alpha-(y \cos \alpha-x \sin \alpha)^{2}=c_{i} \quad i \in \mathbb{Z}, c_{i} \in \mathbb{R}$.

This gives us a system of two equations in $x$ and $y$, whose solutions $(x, y)$ define the locus of the points of coincidence between the two layers. As we have seen above, $c_{i}$ are arbitrary numbers which are identical in both line families; this is the key point which guarantees the correlation between the two layers. Since $c_{i}$ are identical in both line families, we can eliminate them from our two equations. We obtain, therefore,

$$
x \cos \alpha+y \sin \alpha-(y \cos \alpha-x \sin \alpha)^{2}=x-a y^{2}
$$

which gives after some simplifications

$a \sin ^{2} \alpha y^{2}+(\sin \alpha+2 a x \sin \alpha \cos \alpha) y$

$$
-a x^{2} \sin ^{2} \alpha+x(\cos \alpha-1)=0 \text {. }
$$

This second-order equation in $y$ has two solutions:

$$
y=x \tan \frac{\alpha}{2}
$$




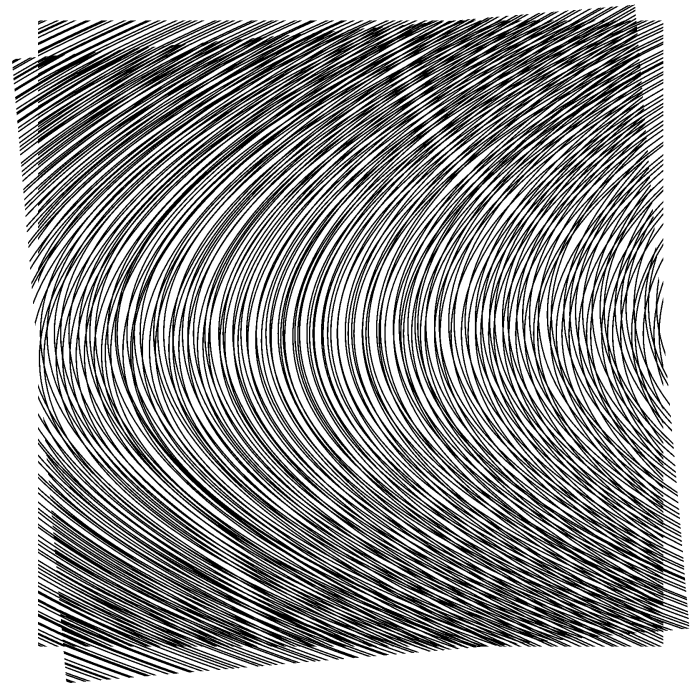

(a)

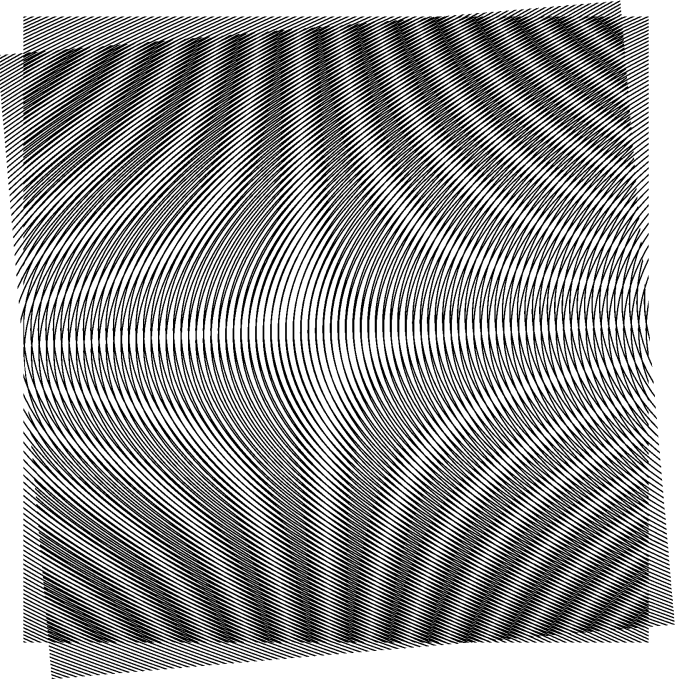

(b)

Figure 10. As figure 7, but with a slight layer shift of $\left(x_{0}, y_{0}\right)=(2 T, 1.5 T)$.

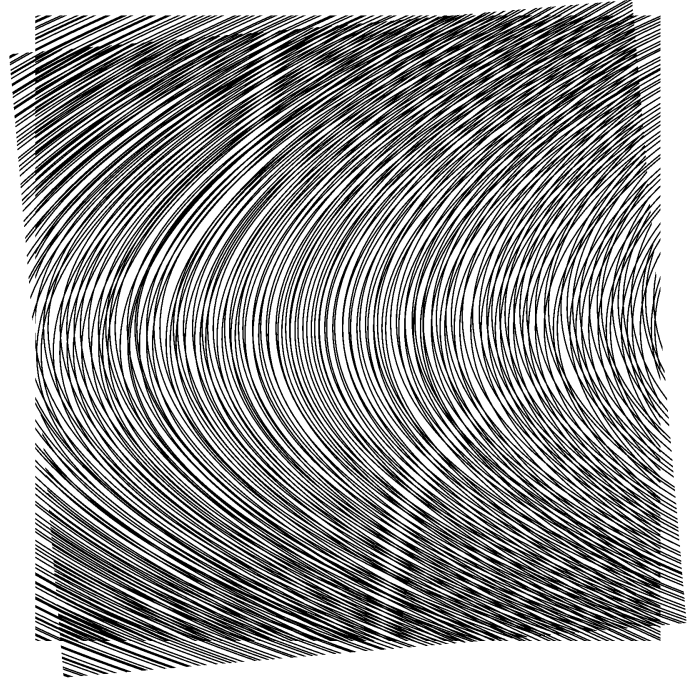

(a)

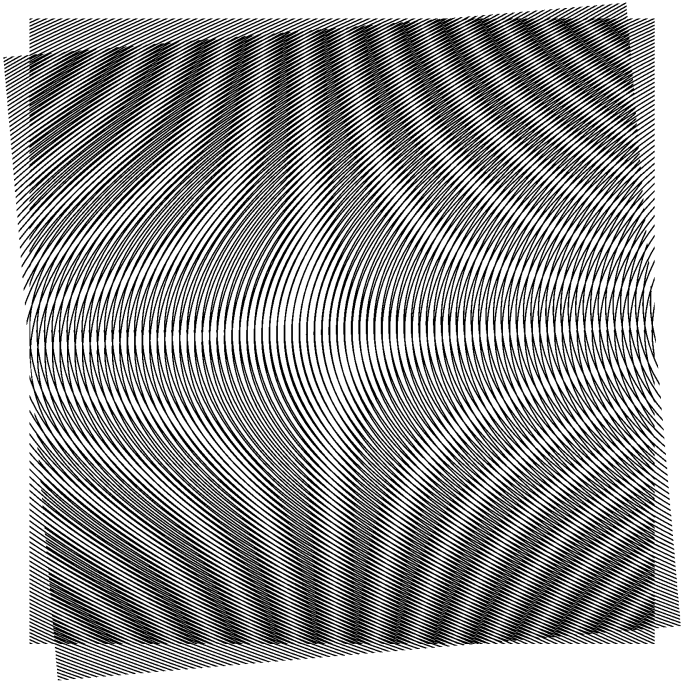

(b)

Figure 11. As figure 7, but with a slight layer shift of $\left(x_{0}, y_{0}\right)=(-T, 1.5 T)$.

and

$$
\begin{aligned}
y & =-x \cot \frac{\alpha}{2}-\frac{1}{a \sin \alpha} \\
& =x \tan \left(90^{\circ}+\frac{\alpha}{2}\right)-\frac{1}{a \sin \alpha} .
\end{aligned}
$$

The solution of our system of equations consists, therefore, of two orthogonal straight lines: one with the angle of $\frac{\alpha}{2}$, passing through the origin, and the other with the angle of $90^{\circ}+\frac{\alpha}{2}$, which crosses the $y$ axis far below the origin, at $y=-\frac{1}{a \sin \alpha}$. These two orthogonal lines are, therefore, the locus of the points of coincidence between the two layers. And indeed, as we can see in figure 7, a Glass pattern is generated in the superposition along these two orthogonal lines. Note that in the repetitive case (figure 7(b)) the arbitrary numbers $c_{i}$ are simply replaced in equations (3) and (4) by integer multiples of the grating periods, $m T$ and $n T$, respectively. As explained in chapter 11 of [3], this gives us the family of hyperbolic moiré bands which are generated in the superposition of the two repetitive gratings. Note that the Glass pattern we have derived above is simply the 0th moiré band of this family, which is obtained when $m=n$ (namely, the moiré band whose index is $p=m-n=0$ ).

What happens now in the superposition when we slightly shift one of the parabolic gratings, say, the unrotated one? A vertical shift of $y_{0}$ will only cause the moiré effects to move horizontally to the right or to the left, depending on the sign of $y_{0}$ (see figures 7,8). However, a horizontal shift of $x_{0}$ has a more interesting influence: as we can see in figures 911 , it causes the locus of coincidence between the two layers to become a hyperbolic curve; and indeed, a Glass pattern is generated in the layer superposition along this curve. But once again, due to the additional ordering which exists in the internal structure of repetitive layers, the superposition in the repetitive case contains, in addition to this Glass pattern, other lines of coincidence between the two layers which are not lines of coincidence in the aperiodic case. This explains the repetitive nature of the moire effect in panels (b) of figures 9-11. 


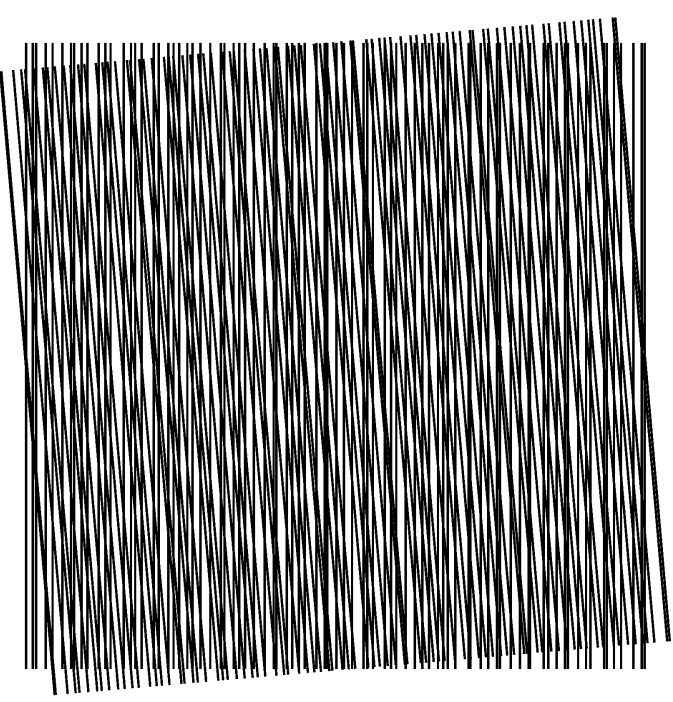

(a)

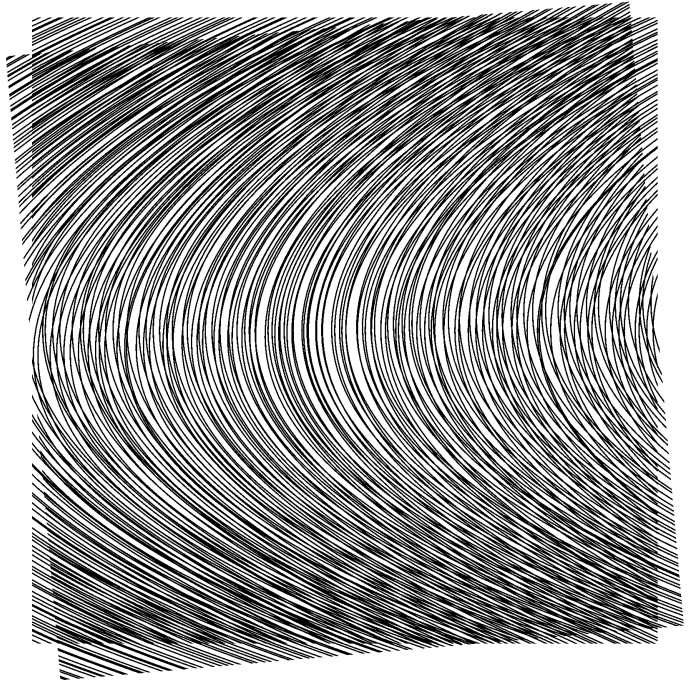

(b)

Figure 12. When the two superposed layers are generated with different seeds for the random number generator, there is no correlation between the two layers and no Glass patterns appear in their superposition. (a) Same as figure 3(a), but with a different seed for each layer. (b) Same as figure 9(a), but with a different seed for each layer.

Looking at this phenomenon the other way around, we recognize here, once again, the 'selectivity' property of the aperiodic superposition (see section 3.3). This means that, depending on the precise layer shifts we choose, the Glass pattern in the aperiodic superposition can extract any desired 'period' from the corresponding repetitive moiré (see figures 8-11).

Finally, it should be stressed that the Glass patterns in aperiodic cases are only generated thanks to the correlation which exists between the superposed layers. As shown in figure 12, if we regenerate the same layer superpositions with a different seed for the random number generator in each layer, there is no correlation between the layers and no Glass patterns appear in the superposition.

\section{Conclusions}

In spite of their different appearance, moiré effects that occur between periodic, repetitive or aperiodic gratings are, in fact, particular cases of the same basic phenomenon, and all of them satisfy the same fundamental rules. The most rudimentary case consists of the superposition of aperiodic (such as random) line gratings. If one of the superposed layers is a rotated, scaled and shifted copy of the other, a Glass pattern appears in their superposition. But unlike in the corresponding case involving random dot screens, the Glass pattern in the case of line gratings is not only generated around an isolated point (the fixed point of the transformation), but it extends into a full line, the line of coincidence between the superposed gratings. This happens because a random line grating is more constrained (i.e. it has fewer degrees of freedom) than a random dot screen, since it only has full freedom in one dimension, while its other dimension is fully determined (constant). Therefore, due to the additional restriction on the structure of the layers (namely, the fact that their second dimension is fully determined by the first one), the layer superposition has a full line of points of coincidence (which obviously includes the fixed point of the underlying mapping).
The corresponding superpositions of periodic or repetitive line gratings, in which the superposed layers undergo the same transformations (rotations, scalings, shifts, etc) as in the aperiodic case, are, in fact, a particular case in which the line distances inside each of the gratings are identical. In this case, in addition to the linear Glass pattern described above, an infinite number of new lines of coincidence (and hence, Glass patterns) is generated throughout the superposition, where the two layers happen to coincide because of the repetitivity in their internal structure. Thus, due to the additional restriction on the structure of the layers (namely, the fact that they are periodic), the layer superposition has a full family of lines of coincidence (which obviously includes the fundamental line of coincidence of the corresponding aperiodic case).

As we can see, in both cases the fact of having additional constraints on the internal structure of the superposed layers causes the addition of new loci of coincidence in the superposition, which are not loci of coincidence in the corresponding superposition of unconstrained layers:

(1) having constrained (fixed) the second dimension of the superposed layers results in the extension of their locus of coincidence from the fixed point of the underlying transformation $\boldsymbol{g}(x, y)$ into a full line; and

(2) adding the constraint of periodicity on the superposed layers causes the extension of this single line of coincidence into a family of infinitely many periodic lines of coincidence.

Looking at property (2) the other way around, we can say that by applying the same layer transformation $\boldsymbol{g}(x, y)$ on aperiodic gratings instead of on periodic gratings, we can select one of the infinitely many repetitions of the Glass patterns which together form the repetitive macroscopic moiré effect. Which one exactly is selected depends on the precise shifts of the original layers, i.e. on their relative positioning. Note, however, that this property concerns only the macrostructures, since the microstructure within these elements may vary 
depending on the case. This interesting result is clearly illustrated by figures 3-6 and 9-11.

Hence, an outstanding property of Glass patterns between aperiodic layers is their macroscopic 'selectivity', i.e. their ability to extract one single macroscopic 'period' from the corresponding repetitive superposition. Moreover, this selectivity can be tuned by means of the relative positioning of the superposed layers: by choosing appropriate layer shifts one can extract in the aperiodic superposition any desired 'period' from the moiré effect in the repetitive superposition.

It should be mentioned that the generalization of our results to cases in which both superposed gratings undergo transformations $\boldsymbol{g}_{1}(x, y)$ and $\boldsymbol{g}_{2}(x, y)$ is straightforward. Also, although we have only mentioned here superpositions of two layers, the results presented in this paper can be easily extended to superpositions of three or more layers.

\section{References}

[1] Glass L 1969 Moiré effect from random dots Nature 223 578-80

[2] Glass L and Pérez R 1973 Perception of random dot interference patterns Nature 246 360-2

[3] Amidror I 2000 The Theory of the Moiré Phenomenon (Dordrecht: Kluwer) 